\title{
Prevalencia de la resistencia genotípica a los fármacos antirretrovirales en pacientes VIH positivos de Tegucigalpa
}

Candy Carbajal, Erika Pérez, Leda Parham,
Ivette Lorenzana de Rivera, Wendy Murillo
Norma Solórzano ${ }^{2}$
Elsa Palou ${ }^{3}$

\section{RESUMEN}

El objetivo de la investigación fue determinar la prevalencia de resistencia del VIH a las drogas antirretrovirales en pacientes tratados y no tratados. Para tal efecto, se analizaron 100 muestras de pacientes $\mathrm{VIH}$ positivos, 50 previos a iniciar tratamiento y 50 bajo tratamiento con evidencia de falla terapéutica, de Tegucigalpa.

Secuencias del gen pol del VIH-1 fueron generadas para determinar la presencia de mutaciones asociadas a resistencia, utilizando la herramienta calibrada para la vigilancia de la resistencia propuesta por la OMS para pacientes pretratados y la lista de mutaciones de la base de datos de Stanford para pacientes bajo tratamiento.

Los resultados muestran que la prevalencia de farmacorresistencia en pacientes previos a iniciar tratamiento fue del $19 \%$ [IC $95 \%$ : 9-33\%], el $8.3 \%$ presentaron mutaciones contra los NRTI, el $12.5 \%$ contra los NNRTI y el $6.25 \%$ contra los PI. Las mutaciones con mayor frecuencia fueron: M184V, K103N, M46I. La prevalencia de mutaciones asociadas a resistencia en los pacientes en falla terapéutica fue del 82 \% [IC $95 \%: 60-90 \%$ ], el $58 \%$ contra los NRTI, $74 \%$ contra los NNRTI y el $22 \%$ contra los PI.

En conclusión, se observó un marcado aumento en la prevalencia de mutaciones asociadas a resistencia en pacientes no tratados, comparada con estudios anteriores, lo cual es de mucha preocupación, ya que limita la eficacia del tratamiento de primera línea. Aunque la prevalencia de resistencia adquirida en

\footnotetext{
${ }^{1}$ Miembros del Grupo de Investigación de Enfermedades Virales en Honduras, beneficiarias de una beca sustantiva de la DICYP. Escuela de Microbología, Facultad de Ciencias, UNAH: wendy.murillo@unah.edu.hn

${ }^{2}$ Instituto Nacional Cardiopulmonar, Tegucigalpa.

${ }^{3}$ Coordinadora de Docencia e Investigación, Hospital Escuela Universitario, Tegucigalpa.
} 
pacientes con falla terapéutica se mantiene ligeramente constante, sigue siendo alta, requiriendo de cambios de tratamiento a segunda o tercera línea en estos pacientes, ocasionando un impacto económico en la salud pública.

Palabras clave: farmacorresistencia, resistencia adquirida, mutaciones, VIH-1.

\section{ABSTRACT}

The objective of this study was to determine the prevalence of HIV drug-resistance in treated and untreated patients. One-hundred samples of HIV-positive patients of Tegucigalpa were analyzed, 50 samples from patients prior to antiretroviral therapy and 50 samples from patients on antiretroviral therapy with treatment failure.

HIV-1 pol sequences were generated to determine the presence of drug resistance mutations using the Stanford calibrated population resistance tool according to the WHO Surveillance Drug Resistance Mutations for untreated patients and the Stanford Database list of mutations for treated patients.

The results shows that drug-resistance mutation prevalence in patients before treatment initiation was $19 \%$ [IC $95 \%$ : 9-33\%], 8.3\% for NRTI, $12.5 \%$ for NNRTI and $6.25 \%$ for PI; the most common mutation founded were: M184V, K103N, M46I. The prevalence of drug-resistance mutations in patients on therapy was $82 \%$ [IC 95 $\%: 60-90 \%$ ], $58 \%$ for NRTI, $74 \%$ for NNRTI, and $22 \%$ for PI.

In conclusions, we observed a significance increase in the prevalence of drug-resistance mutations in untreated patients compared with previous studies, which is of great concern because it limits the effectiveness of first-line treatment. Although the prevalence of acquired resistance in patients with therapeutic failure remains slightly constant remains high, requiring treatment changes to second or third line in these patients, causing an economic impact on public health.

Key words: drug-resistance, acquired resistance, mutations, HIV-1 


\section{INTRODUCCIÓN}

La resistencia del VIH-1 engloba todo un conjunto de mecanismos que el virus puede desarrollar con el fin de soportar y evadir la presión ejercida por determinada droga antirretroviral (ARV) suministrada al paciente infectado. Los virus resistentes a las drogas continúan replicándose en presencia de la droga a la cual se ha hecho resistente. La emergencia de resistencia a las drogas ARV es inevitable en poblaciones que están recibiendo tratamiento $A R V$, aun si el régimen de la terapia es apropiada y si se tiene una adherencia óptima; esto se debe a la naturaleza propia de la replicación del $\mathrm{VIH}-1$, su alta capacidad de mutación en presencia de la presión selectiva de las drogas, a los fenómenos de recombinación viral y a la necesidad de una terapia de por vida.

En general, hay dos tipos de resistencia: adquirida y transmitida. La adquirida ocurre cuando las mutaciones de resistencia son seleccionadas bajo la presión de la droga en pacientes que están recibiendo terapia antirretroviral (TARV). En este tipo de pacientes, la resistencia adquirida puede emerger porque la adherencia es subóptima, por interrupciones del tratamiento, concentraciones inadecuadas de la droga en el plasma, porque el uso de la misma droga es subóptima, por las combinaciones e interacciones entre las drogas o por la naturaleza biológica misma del virus. La resistencia transmitida ocurre cuando individuos son infectados con una cepa viral resistente a las drogas.

En tanto los programas de tratamiento ARV continúan desarrollándose, es imperativo contar con señales de alarma sobre la transmisión de la resistencia del $\mathrm{VIH} \mathrm{a}$ drogas ARV en pacientes previos a iniciar tratamiento, lo cual se observa que también ha aumentado en países de bajos y medianos recursos.

La alta prevalencia de cepas resistentes observadas en las encuestas de monitoreo y los niveles moderados de resistencia transmitida en algunos países, indican que la emergencia y transmisión de cepas virales resistentes están aumentando y que las estrategias para reducir la resistencia deben de implementarse y asegurar el éxito a largo plazo de los programas de tratamiento ARV. La vigilancia de la resistencia permitirá a los países y regiones geográficas rastrear las tendencias de la resistencia del $\mathrm{VIH}$, tomar decisiones racionales y programar actividades de contención en áreas donde sean necesarias, ya sea donde haya un incremento en las prevalencias o una alta prevalencia de resistencia. La vigilancia estandarizada 
de resistencia transmitida o adquirida es crítica para el éxito de la expansión de la TARV en los países en vías de desarrollo.

La Organización Mundial de la Salud (OMS) recomienda estudiar la presencia de cepas virales resistentes a los fármacos ARV en aquellos países en donde el acceso al tratamiento va en aumento, como es el caso de Honduras. Desde que el primer caso de VIH fue diagnosticado en Honduras, en el año 1985, más de 31,823 casos han sido diagnosticados hasta diciembre de 2013.

El Programa Nacional de VIH/SIDA en Honduras ofrece TARV combinada (triterapia) como único tratamiento desde el año 2002. Actualmente 9,569 pacientes están bajo TARV, de estos el $89.2 \%$ están en primera línea, $10.6 \%$ en segunda línea y $0.2 \%$ están con terapia de rescate.

Pocos estudios han sido dirigidos a evaluar la prevalencia de resistencia a las drogas ARV en Honduras. En 2010 se reportó por primera vez una prevalencia de resistencia adquirida del $81 \%$ en un grupo de pacientes con falla terapéutica, el estudio fue realizado con muestras colectados entre 2004-2007. Estudios realizados internacionalmente han reportado $80 \%$ de resistencia adquirida en una cohorte de pacientes con falla terapéutica en Inglaterra, $76 \%$ en EE.UU. y $88 \%$ en Francia. Los resultados hondureños son comparables con los resultados de otros países, a pesar de que en estos países las condiciones de TARV son bastante diferentes.

En la región centroamericana existe poca información con respecto a la resistencia transmitida. En un estudio dirigido a investigar la epidemiología molecular del VIH en seis países centroamericanos con muestras colectadas entre el 2002-2010, se pudo evaluar la prevalencia de resistencia a las drogas ARV; este estudio reportó una prevalencia global de resistencia adquirida del $62 \%$ y una prevalencia global de resistencia transmitida del $7.5 \%$. Lloyd y colaboradores (2008) reportaron una prevalencia de resistencia transmitida del $9.2 \%$ en pacientes hondureños que iban a iniciar tratamiento bajo el Programa Nacional de TARV durante el periodo 20022003. Recientemente se reportó una prevalencia de resistencia transmitida del $7 \%$ en pacientes vírgenes al tratamiento (naïve) en población captada durante el 20042007.

Es de gran importancia estudiar la presencia de resistencia a las drogas ARV en pacientes que van a iniciar el tratamiento, ya que la presencia de cepas resistentes 
pueden limitar las opciones de tratamiento en el futuro. De igual manera, es importante estudiar la presencia de resistencia en pacientes tratados con falla terapéutica, ya que conociendo los perfiles de resistencia se les puede ofrecer a estos mejores alternativas de tratamiento para que este sea eficaz.

Los resultados generados en este tipo de estudios son de gran relevancia para los pacientes por lo antes mencionado y para el Programa Nacional de VIH/SIDA, ya que orienta la elaboración de las políticas/guías de tratamiento en Honduras; adicionalmente puede optar por medidas preventivas en conjunto con otras acciones 0 indicadores de alerta temprana. Al mismo tiempo, aportan nuevos conocimientos para la comunidad científica en general. Por lo anteriormente expuesto, el objetivo del presente estudio fue determinar la presencia de mutaciones asociadas a resistencia en pacientes infectados con VIH-1, bajo TARV, con falla terapéutica y en pacientes previos a iniciar tratamiento.

\section{METODOLOGÍA}

\section{Población de estudio}

La población de estudio consistió de 100 pacientes VIH-1 positivos atendidos en los Centros de Atención Integral (CAI) del Hospital Escuela Universitario (CAI-HEU) y del Instituto Nacional Cardiopulmonar (CAI-INCP), de Tegucigalpa. Fueron incluidos en el estudio aquellos pacientes $\mathrm{VIH}-1$ positivos confirmados laboratorialmente bajo los criterios utilizados por el algoritmo nacional para el diagnóstico de la infección por $\mathrm{VIH}-1$ e identificados por los médicos tratantes en dos grupos: 1) cincuenta pacientes $\mathrm{VIH}-1$ positivos previos a iniciar TARV, 25 procedentes de cada CAl; y 2) cincuenta pacientes VIH-positivos tratados con evidencia de falla terapéutica virológica, inmunológica o clínica, 25 procedentes de cada CAl.

Siguiendo las consideraciones éticas, el estudio fue aprobado por el Comité de Ética en Investigación Biomédica de la Unidad de Investigación Científica, Facultad de Ciencias Médicas, UNAH; y los participantes incluidos en el estudio llenaron un consentimiento informado. 


\section{Colección y procesamiento de las muestras}

Alos pacientes que accedieron a participar en el estudio se les tomó una muestra de sangre total, entre $5-7 \mathrm{ml}$, en tubos estériles con anticoagulante EDTA. Las muestras fueron colocadas en los CAl seleccionados para el estudio y transportadas en cadena de frío al laboratorio de virología de la UNAH para su procesamiento el mismo día de la toma de la muestra.

Las muestras de sangre total se procesaron mediante centrifugación para realizar la separación del plasma y linfocitos. Las muestras, una vez procesadas, fueron utilizadas para la extracción del material genético (ARN), mediante el método QIAmp ARN (QIAGEN®, Santa Clara, California, EE.UU.), siguiendo las instrucciones del fabricante. Posteriormente, se realizó la síntesis del ADN complementario (cADN) mediante el protocolo ThermoScript ${ }^{T M}$ RT-PCR (Invitrogen $^{\mathrm{TM}}$ Life Technologies ${ }^{\mathrm{TM}}$ ). El análisis genotípico para determinación de la resistencia fue realizado utilizado el cADN para amplificar una región del gen pol del $\mathrm{VIH}-1$ (proteasa y la primera mitad de la transcriptasa reversa) mediante un protocolo de secuenciación "in-house" previamente publicado.

Una vez obtenidas las secuencias, se realizó la revisión y análisis de las mismas para investigar mutaciones asociadas a resistencia y la susceptibilidad a las drogas antirretrovirales, utilizando la lista de mutaciones para la vigilancia de la resistencia del VIH-1 a las drogas ARV de 2009, propuesta por la OMS, usando la herramienta calibrada para la determinación de resistencia transmitida que pone a disposición la base de datos de VIH de la Universidad de Stanford en línea (Calibrated Population Resistance Tool vs 6.0), para las secuencias de los pacientes sin tratamiento previo.

Para los pacientes con falla terapéutica se utilizó la lista de mutaciones de la base de datos de VIH de la Universidad de Stanford para pacientes bajo tratamiento antirretroviral. La base de datos de la Universidad de Stanford también proporcionó un protocolo para cada una de las muestras especificando la susceptibilidad 0 resistencia a las drogas antirretrovirales (contra proteasa o transcriptasa reversa).

\section{Análisis estadístico}

Los datos se analizaron utilizando el programa estadístico VassarStats. Las prevalencias encontradas, intervalos de confianza del $95 \%$ y los valores $p$, fueron calculados usando distribución binomial y el método exacto. 


\section{RESULTADOS}

\section{Características de la población de estudio}

De los 100 pacientes incluidos en el estudio, el $42 \%$ eran del sexo femenino y el $58 \%$ del sexo masculino; con un promedio de edad de 39 años, con un rango de 17-73 años. En cuanto a la vía de transmisión, la mayoría, un $90 \%$, adquirió la infección por transmisión heterosexual; $7 \%$ por transmisión homosexual (hombres que tienen sexo con hombres, $\mathrm{HSH}$ ), $1 \%$ por transmisión bisexual, $1 \%$ transmisión madre a hijo $(\mathrm{TMH})$ y un $1 \%$ reportó transmisión por transfusión sanguínea.

El promedio del conteo de linfocitos CD4+ fue de $236 \mathrm{cel} / \mathrm{mm}^{3}$ con un rango de 7 $1434 \mathrm{cel} / \mathrm{mm}^{3}$; el promedio de carga viral fue de $421,085 \mathrm{copias} / \mathrm{ml}$ con un rango de 39-13200000 copias/ml. Con base a la sintomatología que presentaban los pacientes, se clasificaron en tres estadios clínicos: I: asintomático, II: con sintomatología no definitoria de SIDA y III: pacientes con síntomas de SIDA; el 80 $\%$ mostró estar en estadio clínico I; 9 \% en estadio clínico II y $11 \%$ en estadio clínico III. Las características generales de los pacientes por grupo de estudio, pacientes tratados y no tratados están resumidas en la tabla 1.

De las 100 muestras analizadas por secuenciación, 98 fueron secuenciadas exitosamente, 48 de pacientes no tratados y 50 de pacientes tratados.

Tabla 1. Características generales de los pacientes incluidos en el estudio, por centro y grupo de estudio

\begin{tabular}{|c|c|c|c|c|c|}
\hline \multirow[b]{2}{*}{ Descripción } & \multicolumn{2}{|c|}{ CAI-INCP } & \multicolumn{2}{|c|}{ CAI-HEU } & \multirow[b]{2}{*}{ Total } \\
\hline & $\begin{array}{l}\text { Pacientes } \\
\text { no tratados }\end{array}$ & $\begin{array}{c}\text { Pacientes } \\
\text { tratados }\end{array}$ & $\begin{array}{c}\text { Pacientes } \\
\text { no tratados }\end{array}$ & $\begin{array}{c}\text { Pacientes } \\
\text { tratados }\end{array}$ & \\
\hline No. de pacientes & 25 & 25 & 25 & 25 & 100 \\
\hline $\begin{array}{l}\text { Sexo } \\
\text { Femenino }\end{array}$ & 8 & 12 & 8 & 14 & 42 \\
\hline Masculino & 17 & 13 & 17 & 11 & 58 \\
\hline $\begin{array}{l}\text { Edad (en años) } \\
\text { Promedio } \\
\text { Ranan }\end{array}$ & $\begin{array}{c}37 \\
18-55\end{array}$ & $\begin{array}{c}43 \\
28-62\end{array}$ & $\begin{array}{c}37 \\
19-73\end{array}$ & $\begin{array}{c}38 \\
17-56\end{array}$ & $\begin{array}{c}39 \\
17-73\end{array}$ \\
\hline $\begin{array}{l}\text { Riesgo de transmisión } \\
\text { Heterosexual }\end{array}$ & 21 & 24 & 23 & 22 & 90 \\
\hline Homosexual & 4 & 1 & - & 2 & 7 \\
\hline Bis & & - & 1 & - & 1 \\
\hline
\end{tabular}




\begin{tabular}{|l|c|c|c|c|c|}
\hline \multirow{2}{*}{ Descripción } & \multicolumn{3}{|c|}{ CAI-INCP } & \multicolumn{2}{c|}{ CAI-HEU } \\
\cline { 2 - 6 } & $\begin{array}{c}\text { Pacientes } \\
\text { no tratados }\end{array}$ & $\begin{array}{c}\text { Pacientes } \\
\text { tratados }\end{array}$ & $\begin{array}{c}\text { Pacientes } \\
\text { no tratados }\end{array}$ & $\begin{array}{c}\text { Pacientes } \\
\text { tratados }\end{array}$ & Total \\
\hline \begin{tabular}{l|c|c|c|c|c|}
\hline Parenteral \\
Madre-hijo
\end{tabular} & - & - & 1 & - & 1 \\
\hline $\begin{array}{l}\text { Conteo de CD4 (células/ul) } \\
\text { Promedio }\end{array}$ & 288 & - & - & 1 & 1 \\
\hline $\begin{array}{l}\text { Rango } \\
\text { Carga viral (copias/ml) }\end{array}$ & $7-789$ & $21-511$ & $16-1434$ & $7-882$ & $7-1434$ \\
\hline $\begin{array}{l}\text { Promedio } \\
\text { Rango }\end{array}$ & 905,874 & 110,420 & 636,231 & 48,865 & 445,348 \\
\hline $\begin{array}{l}\text { Estadío clínico } \\
1\end{array}$ & $129-$ & $57-$ & $39-$ & $103-$ & $39-$ \\
\hline 2 & $13,200,000$ & 696,941 & $9,992,770$ & 447,585 & $13,200,000$ \\
\hline 3 & 18 & 19 & 23 & 22 & 82 \\
\hline
\end{tabular}

Resistencia del VIH-1 a los fármacos ARV en pacientes previo a iniciar tratamiento

Mutaciones asociadas a resistencia fueron encontradas en 9 de los 48 pacientes previos a iniciar TARV, presentando una prevalencia total del $19 \%$ [IC 95\%: 9-33 $\%$ ]. La prevalencia de la resistencia a los medicamentos de acuerdo a la clase de drogas fue del $12.5 \%$ (6/48) [IC $95 \%$ : 5-26\%] para los inhibidores no nucleósidos de la transcriptasa reversa (NNRTI), $8.3 \%$ (4/48) [IC 95\%:3-20\%] para los inhibidores nucleósidos de la transcriptasa reversa (NRTI) y $6.25 \%$ (3/48) [IC $95 \%: 2-17 \%$ ] para los inhibidores de la proteasa (PI). El 56 \% (5/9) presentaron mutaciones asociadas a resistencia a una sola clase de drogas, 60 $\%(3 / 5)$ contra los NNRTI y $40 \%$ (2/5) contra los PI; un $44 \%$ (4/9) contra dos clases de drogas, $75 \%$ (3/4) para la combinación NRTI + NNRTI y $25 \%$ (1/4) para NRTI + PI. La tabla 2 muestra un resumen de las mutaciones asociadas a resistencia encontradas en esta población de estudio. 
Tabla 2. Características de los 9 pacientes previos a iniciar TAVR con mutaciones asociadas a resistencia

\begin{tabular}{|c|c|c|c|c|c|c|c|c|}
\hline \multirow{2}{*}{ No. } & \multirow{2}{*}{ CAI } & \multirow{2}{*}{$\begin{array}{c}\text { Géne- } \\
\text { ro }\end{array}$} & \multirow{2}{*}{$\begin{array}{c}\text { Edad } \\
\text { (años) }\end{array}$} & \multirow{2}{*}{ Transmisión } & \multirow{2}{*}{$\begin{array}{l}\text { Tiempo de } \\
\text { diagnós- } \\
\text { tico (días) }\end{array}$} & \multicolumn{3}{|c|}{ Mutaciones asociadas a resistencia } \\
\hline & & & & & & NRTI & NNRTI & PI \\
\hline 1 & INCP & M & 30 & Homosexual & 36 & & K103N & \\
\hline 2 & INCP & M & 55 & Heterosexual & 11 & & K103N & \\
\hline 3 & INCP & $\mathrm{F}$ & 49 & Heterosexual & 660 & M184V & $\begin{array}{l}\text { K103N, } \\
\text { V108I }\end{array}$ & \\
\hline 4 & INCP & M & 23 & Heterosexual & 3600 & & & $185 \mathrm{~V}$ \\
\hline 5 & INCP & M & 46 & Heterosexual & 13 & & F227FL & \\
\hline 6 & HEU & $\mathrm{F}$ & 26 & Heterosexual & 10 & T215S & $\mathrm{P} 225 \mathrm{H}$ & \\
\hline 7 & HEU & M & 64 & Heterosexual & 4 & $\begin{array}{l}\text { M184V, M41L } \\
\text { T215F, D67G }\end{array}$ & & $\begin{array}{l}\text { M46I, 154V, } \\
185 \mathrm{~V}, 150 \mathrm{~V}\end{array}$ \\
\hline 8 & HEU & M & 20 & Heterosexual & 450 & M184V,T215S & K101E, & \\
\hline 9 & HEU & M & 22 & Bisexual & 18 & & G190S & M46I \\
\hline
\end{tabular}

Resistencia del VIH-1 a los fármacos ARV en pacientes tratados con falla terapéutica

La prevalencia de mutaciones asociadas a resistencia a los fármacos ARV en pacientes tratados con evidencia de falla terapéutica fue del $82 \%$ [IC 95\%:60-90 \%]. Se observó una prevalencia del 58 \% (29/50) [IC $95 \%: 44-71 \%$ ] contra los NRTI, 74 \% (37/50) [IC $95 \%: 60-84 \%$ ] contra los NNRTI y un $22 \%$ (11/50) [IC $95 \%: 13-35 \%$ ] contra los PI. En cuanto a las combinaciones de fármacos ARV, se observó que el 73 $\%$ (30/41) presentó mutaciones asociadas a resistencia contra dos o más clases de drogas ARV, de estos, el $40 \%$ (20/50) presentaba mutaciones contra los NRTI + NNRTI, un $2 \%$ contra los NRTI + PI, $6 \%$ (3/50) contra los NNRTI + PI y un $12 \%(6 / 50)$ contra las tres clases de fármacos ARV.

Del total de pacientes de este grupo de estudio, el $72 \%$ (36/50) habían tenido un solo esquema de tratamiento, $24 \%(12 / 50)$ dos esquemas de tratamiento y el $4 \%(2 / 50)$ tres esquemas de tratamiento. El $64 \%$ estaba en la primera línea de tratamiento, siendo los esquema $A Z T+3 T C+E F V, A Z T+3 T C+N V P$ y $A Z T+3 T C+A B C$ los más frecuentes y un $36 \%(18 / 50)$ estaba bajo la segunda línea de tratamiento, siendo ABC+LPV/r+TDF el esquema más frecuente con un $28 \%(5 / 18)$. El $100 \%(50 / 50)$ presentó falla virológica, un $94 \%$ (47/50) falla inmunológica y $26 \%$ (13/50) falla clínica.

De los pacientes con resistencia, el $24 \%$ (10/41) estaba en el segundo y tercer 
esquema de tratamiento, con un promedio de recuento de linfocitos CD4+ de 166 células $/ \mathrm{mm}^{3}$ y una carga viral promedio de 80,139 copias $/ \mathrm{ml}$. El $20 \%(8 / 41)$ presentaba sintomatología, $50 \%$ (4/8) estadio clínico II y $50 \%$ (4/8) estadio clínico III.

\section{DISCUSIÓN}

Este estudio revela resultados recientes sobre la prevalencia de transmisión de cepas resistentes en pacientes previos a iniciar tratamiento $A R V$ y la prevalencia de resistencia adquirida a las drogas ARV en pacientes bajo tratamiento con evidencia de falla terapéutica. Los resultados muestran un marcado aumento en la prevalencia de mutaciones asociadas a resistencia en pacientes previos a iniciar tratamiento, del $6.5 \%$ reportada por Lloyd y colaboradores (2008), comparada con el $19 \%$ observada en este estudio [OR=3.3; IC 95\%:1.3-8.1].

Según la clasificación de la OMS, la prevalencia de mutaciones asociadas a resistencia ha pasado de moderada a alta. La prevalencia de resistencia adquirida en pacientes con falla terapéutica (82\%) se mantiene similar a la reportada en el 2010.

Al contar con más de diez años de iniciada la TARV combinada en el país, se esperaría un ligero incremento en la prevalencia de la transmisión de cepas resistentes a los fármacos ARV como consecuencia del mayor acceso a la terapia, como se ha observado en aquellos países en donde el acceso al tratamiento ARV ha sido más amplio. De igual manera, con el paso del tiempo se esperaría observar un incremento en la prevalencia de transmisión de mutaciones contra drogas que anteriormente reportaban una baja prevalencia, como es el caso de los inhibidores de proteasa que el presente estudio reporta con una prevalencia del $6.25 \%$ y en el estudio de Lloyd reportaba una prevalencia del $1.4 \%$ (Lloyd, 2008). También es motivo de preocupación observar que casi la mitad de los pacientes con resistencia (44\%) presentan mutaciones contra dos clases de drogas, limitando las futuras opciones de tratamiento para estos pacientes.

La mayoría de los pacientes que no habían iniciado TARV tenían un diagnóstico menor de 5 meses y no presentaban sintomatología al momento de la toma de 
muestra. Se observa que la transmisión heterosexual sigue siendo la principal vía de transmisión de la infección en ambos grupos de estudio. La mayoría de los pacientes bajo tratamiento reportaban falla virológica e inmunológica, $100 \%$ y $96 \%$, respectivamente; aunque esta condición aún no estuviera causando sintomatología en ellos, ya que solo un $26 \%$ presentó falla clínica.

En el grupo de pacientes tratados con falla terapéutica se observa una considerable disminución en las prevalencia de mutaciones asociada a resistencia contra los NRTI (58 \%) y contra los PI (22\%), comparados con las prevalencias reportadas previamente, $71 \%$ y $37 \%$, respectivamente; esto puede deberse a que en el estudio anterior un $42 \%$ de los pacientes que habían iniciado con mono y biterapia, a diferencia del presente grupo de estudio, que todos estaban bajo terapia antirretroviral combinada (triple terapia). Por el contrario, se observa un ligero aumento en la prevalencia de mutaciones asociada a resistencia contra los NNRTI (74 \%) comparada con la reportada previamente $(70 \%)$, esto puede explicarse porque la barrera genética de los NNRTI es baja y una sola mutación puede comprometer la susceptibilidad a todas las drogas de esta clase.

El $60 \%$ de los pacientes tratados con resistencia antirretroviral tenían mutaciones contra dos o más clases de drogas, la combinación más frecuente de drogas ARV fue contra los NRTI + NNRTI (40\%, IC $95 \%$ : 27-55\%), que es la combinación que incluye el esquema de primera línea de tratamiento que se utiliza en el país, lo cual llama la atención, porque significa que entre un $27 \%$ y $55 \%$ de los pacientes tienen que cambiar la primera línea de tratamiento por desarrollar mutaciones asociadas a resistencia contra estas dos clases de drogas.

Otro dato importante de resaltar es que el $12 \%$ [IC $95 \%$ : 6-24 \%] de los pacientes con tratamiento presentan mutaciones contra las tres clases de drogas disponibles en el país, indicando que estos pacientes necesitan terapia de rescate, limitando las opciones de tratamiento para el paciente, porque este tipo de terapia no está disponible en el programa nacional, sino que se tiene que recurrir a donaciones de organizaciones internacionales o se incurre en costos para el paciente, ya que tiene que comprarlos a nivel privado. 


\section{CONCLUSIONES}

De los pacientes que no habían iniciado tratamiento, un $19 \%$ presentó mutaciones asociadas a resistencia (presencia de resistencia transmitida), lo cual es de mucha preocupación porque limita las opciones de tratamiento para estos pacientes. En comparación con estudios anteriores, la resistencia transmitida en este tipo de pacientes se ha incrementado casi el triple, comparado con el $6.5 \%$ reportado por Lloyd (2008) en pacientes previo a iniciar tratamiento y más del doble, $7 \%$, reportado por nuestro grupo en pacientes recién diagnósticados que no habían recibido tratamiento. Ademas, de acuerdo a la clasificación de la resistencia transmitida de la OMS, este porcentaje se considera alto y las acciones a tomar con resultados así son una revisión en los esquemas de tratamiento, específicamente de primera línea y continuar realizar estudios de este tipo cada año.

Los pacientes bajo tratamiento ARV con falla terapéutica presentaron una prevalencia del $82 \%$ a alguna mutación asociada con resistencia, lo cual no es sorprendente, porque ya había al menos un signo de falla al tratamiento (inmunológica, virológica o clínica) y por eso se realizó la genotipificación. Lo importante en estos pacientes es que con el resultado de resistencia (genotipificación), los médicos tratantes pueden buscar mejores opciones para su tratamiento.

Con respecto a la presencia de mutaciones asociadas a resistencia a las diferentes drogas ARV, los NNRTI y los NRTI siguen siendo las más altas, $13 \%$ y $8 \%$ en los pacientes no tratados y del $74 \%$ y $58 \%$ en los pacientes tratados, respectivamente. Estos resultados son de mucha preocupación, ya que estas dos clases de drogas son las utilizadas como primera línea de tratamiento, por lo que el Programa Nacional de VIH/SIDA debe valorar cuáles son las causales de este incremento y tomar acciones que en conjunto con los indicadores de alerta temprana conlleve a un mejor manejo del paciente previo y bajo terapiaARV.

El mayor beneficio de esta investigación será para los pacientes participantes, ya que los resultados generados a través de la misma servirán para orientar a los médicos tratantes sobre las mejores opciones de tratamiento para cada paciente en particular. 


\section{AGRADECIMIENTOS}

A los médicos tratantes de los Centros de Atención Integral del Instituto Nacional Cardiopulmonar y del Hospital Escuela Universitario por su valiosa colaboración en la identificación e invitación de los pacientes para participar en el estudio; asimismo, a todos los participantes del estudio. Gracias a la Dirección de Investigación Científica y Posgrado de la UNAH por haber auspiciado esta investigación.

\section{BIBLIOGRAFÍA}

Bennett, Diane. et al. (2008). The World Health Organization's global strategy for prevention and assessment of HIV drug resistance. Antiviriral Therapy, 13 Supplement 2, 1-13.

Casado, José L. et al. (2000). Non-nucleoside reverse transcriptase inhibitor resistance among patients failing a nevirapine plus protease inhibitor-containing regimen. AIDS, 14(2), F1-7.

Clavel, F. \& Hance, A. J. (2004). HIV drug resistance. New England Journal Medecine, 350(10), 1023-1035.

Costagliola, Dominique et al. (2007). Prevalence of HIV-1 drug resistance in treated patients: a French nationwide study. Journal of Acquired Immune Deficiency Syndrome, 46(1), 12-18.

Frentz, Dineke. et al. (2014). Patterns of Transmitted HIV Drug Resistance in Europe Vary by Risk Group. PLoS One, 9(4), e94495.

Gupta, Ravindra. K. et al. (2000). Global trends in antiretroviral resistance in treatment-naive individuals with HIV after rollout of antiretroviral treatment in resource-limited settings: a global collaborative study and meta-regression analysis. Lancet, 380(9849), 1250-1258.

Hammer, Scott. M. et al. (2008). Antiretroviral treatment of adult HIV infection: 2008 recommendations of the International AIDS Society-USA panel. JAMA, 300(5), 555-570.

Joly, Veronique. et al. (2000). Delavirdine in combination with zidovudine in treatment of human immunodeficiency virus type 1 -infected patients: evaluation of efficacy and emergence of viral resistance in a randomized, comparative phase III trial. The M/3331/0013B Study Group. Antimicrobisl Agents Chemotherapy, 44(11), 3155-3157. 
Lloyd, Bradley. et al. (2008). Prevalence of resistance mutations in HIV-1-Infected Hondurans at the beginning of the National Antiretroviral Therapy Program. AIDS Research and Human Retroviruses, 24(4), 529-535.

Murillo, Wendy. et al. (2010). Prevalence of drug resistance and importance of viral load measurements in Honduran HIV-infected patients failing antiretroviral treatment. HIV Medicine, 11(2), 95-103.

Murillo, Wendy. et al. (2010). Transmitted drug resistance and type of infection in newly diagnosed HIV-1 individuals in Honduras. Journal of Clinical Virology, 49(4), 239-244.

Murillo, Wendy. et al. (2013). A single early introduction of HIV-1 subtype B into Central America accounts for most current cases. Journal of Virology, 87(13), 7463-7470.

Pillay, Deenan. et al. (2005). Estimating HIV-1 drug resistance in antiretroviraltreated individuals in the United Kingdom. Journal of Infectious Diseases, 192(6), 967-973.

Reporte Nacional de VIH/SIDA. (2014). Tegucigalpa, Honduras: Programa Nacional de ITS/VIH/SIDA. Secretaría de Salud de Honduras.

Richman, Douglas. D. et al. (2004). The prevalence of antiretroviral drug resistance in the United States. AIDS, 18(10), 1393-1401.

Shafer, Robert. W. et al. (2007). HIV-1 protease and reverse transcriptase mutations for drug resistance surveillance. AIDS, 21(2), 215-223. 